\title{
Clinico-etiological profile of patients presenting with hemoptysis diagnosed by fiber optic bronchoscopy - A study done in tertiary care centre of central Karnataka
}

\author{
Kushal Bondade ${ }^{1}$, Eti Ajit ${ }^{2 *}$, Hemanth K. Kulkarni ${ }^{3}$, Antonio L. Dacosta ${ }^{4}$, Mario Pinto ${ }^{5}$ \\ ${ }^{1,2,5}$ Assistant Professor, ${ }^{3}$ Associate Professor, ${ }^{4}$ Professor, S.S. Institute of Medical Sciences and Research Centre, Davanagere, \\ Karnataka, India
}

*Corresponding Author:

Email: ajith.eti1@gmail.com

\begin{abstract}
Introduction: Hemoptysis is a common complaint among patients attending chest clinics. Clinical profile and etiology may vary depending upon patient population studied. Fiber optic bronchoscopy (FOB) is a useful diagnostic tool in such cases to establish accurate diagnosis.

Aim:

1. To study the clinical and etiological profile of patients presenting with hemoptysis at our tertiary care centre.

2. To define the diagnostic yield of FOB in these patients.

Materials and Methods: Institutional based observational prospective study was conducted on 60 patients who presented with hemoptysis to the department of pulmonary medicine at our tertiary care centre. All patients underwent FOB after thorough clinical, radiological and laboratory evaluation.

Results: Mean age of the patients studied was $43.93 \pm 13.20 \mathrm{yrs}$, predominant were male $38(63.3 \%)$. Sputum production in 42 patients $(70 \%)$ was the most common associated symptom followed by dyspnea 34(56.7\%) and fever 20(33.3\%). On FOB, congestion of the endobronchial lumen was seen in 44 (73.3\%), ectasia in 16 (26.6\%), bleeding site was localized in $30(50 \%)$ patients. Broncho alveolar lavage (BAL) was positive for Acid fast bacilli in 12 (20\%), bacterial culture in 6 (10\%) and fungal elements in $2(3.33 \%)$ patients. Endobronchial biopsy (EBB) diagnosed lung malignancy in 10(16.7\%) patients.

Conclusion: Bronchiectasis was the most common cause of hemopytsis followed by pulmonary tuberculosis. FOB resulted in diagnosis of three-fourths of the patients analysed in our study with a positive yield of $76.6 \%$.
\end{abstract}

Keywords: Hemoptysis, Etiological profile, Fiberoptic bronchoscopy (FOB).

\section{Introduction}

Hemoptysis refers to expectoration of blood from the tracheobronchial tree. It ranges from mere blood streaking of sputum to expectorating massive volume of blood. Hemoptysis of any volume implies a life threatening process which mandates immediate evaluation and treatment[1]. Hemoptysis is a chief complaint in $7-15 \%$ of patients attending respiratory clinics.[2].

Common causes for hemoptysis in India include pulmonary tuberculosis (TB), malignancy, bronchiectasis, chronic bronchitis, bacterial pneumonia, fungal infections but it could be a manifestation of more than 100 clinical conditions.[3] However the clinical presentation and etiological profile of hemoptysis may differ based on socio-economic, demographic and behavioural parameters. Hence this study was taken up to know the clinical and etiological pattern for hemoptysis in central part of Karnataka.

Around $30 \%$ of hemoptysis cases pose difficult diagnostic problem and may not show any identifiable etiology despite thorough evaluation.[4] These patients routinely undergo proper history and clinical evaluation, sputum analysis, imaging like chest x-ray (CXR) and computed tomography (CT) thorax. In resource poor setting CT thorax may not be feasible.

Fiberoptic bronchoscopy (FOB) is a safe and minimally invasive diagnostic tool performed under local anesthesia or conscious sedation. It provides direct visualization of the tracheobronchial tree to anatomically localize the bleeding site. Further sampling of respiratory tract done by bronchoalveolar lavage (BAL) and endobronchial biopsy (EBB) improves diagnostic yield considerably in most cases. Thus the second objective of our research was to define the role and diagnostic yield of FOB in undiagnosed hemoptysis.

\section{Materials and Methods}

This observational prospective study was carried out in the department of pulmonary medicine of SS Institute of medical sciences and research centre, Davanagere between April 2017 and March 2018. Institutional clearance was obtained from the Ethical and Research Committee. Patients aged more than 18 yrs having undiagnosed hemoptysis with normal or abnormal CXR, patients with preliminary diagnosis of pulmonary TB and malignancy based on clinicoradiological findings were included in the study. Patients with moderate to massive life threatening hemoptysis, patients with unstable cardiovascular status, neurological disorders, patients with severe bronchospasm and hypoxemia, bleeding diathesis were excluded. FOB was performed after taking informed consent under conscious sedation plus topical anesthesia. BAL and EBB was taken as indicated and 
were subjected for relevant microbiological and pathological investigations. Data obtained was entered in excel sheet and further analysed.

\section{Results}

A total of 60 patients were included in the study after considering inclusion and exclusion criteria. 38 patients $(63.3 \%)$ were male and $22(36.7 \%)$ were female. The age group among the patients studied varied from 21 to 70 years with mean age of $43.93 \pm 13.20$ and most of them falling into the age group of 40-59 years. Diabeties mellitus was present in 4(6.7\%) and hypertension in 10 patients (16.7\%). Past history of pulmonary TB was present in 26(43.3\%) of the cases. Tobacco smoking was present in 26(43.3\%) and tobacco chewing in $8(13.3 \%)$. (Table no.1)

CXR was normal in 12 patients (20\%). Among 48 patients with abnormal CXR, 16(26.7\%) had bronchiectasis, fibrocavitations $10(16.6 \%)$, mass lesion $8(13.3 \%)$, unresolved pneumonia 6(10\%), hilar adenopathy $4(6.7 \%)$ and collapse $2(3.3 \%)$ Sputum for Acid fast bacilli (AFB) was negative in all patients before undergoing FOB. Sputum production in 42 patients $(70 \%)$ was the predominant associated symptom followed by dyspnea $34(56.7 \%)$ and fever 20(33.3\%) (Table 2)

Gross bronchoscopic examination revealed congestion/hyperaemia of the endobronchial lumen in $44(73.3 \%)$ of the patients which was the most common finding followed by active bleeding in 30(50\%) of the cases. Ectasia of the endobronchial lumen was present in $16(26.6 \%)$, endobronchial growth was seen in $10(16.6 \%)$ patients and endobronchial ulcer/erosion was seen in $4(6.7 \%)$ of the patients. (Table 3 )

BAL analysis was positive for AFB in 12(20\%), growth on bacterial culture was seen in $6(10 \%)$ and positive for fungal elements in 2(3.33\%) patients. EBB was taken in the presence of growth visualized during the procedure. Histopathological examination (HPE) of the obtained specimen revealed squamous cell carcinoma in 6 patients $(10 \%)$ and adenocarcinoma in 4 patients $(6.7 \%)$. (Table 4 )

Among 60 patients with hemoptysis who underwent FOB at our centre, bronchiectasis 16(26.7\%) was the most common cause followed by active pulmonary TB $12(20 \%)$ and lung cancer $10(16.7 \%)$. Bacterial pneumonia was diagnosed in 6 patients $(10 \%)$ and fungal infection in 2 (3.33\%). However active pathology was not detected in $14(23.3 \%)$ of the patients. FOB resulted in definitive diagnosis in threefourth of the patients analysed in our study with a positive yield of $76.6 \%$.(Table no.5)

\section{Discussion}

Hemoptysis is the cheif presenting complaint in about $10-15 \%$ of the patients attending pulmonology $\mathrm{OPD}[2]$. It is a manifestation of wide spectrum of pulmonary diseases and is challenging for the treating physician to establish the etiology. The etiological possibilities of hemoptysis may vary depending upon patient population studied. We studied the clinical profile, CXR findings, FOB findings, etiological trend of hemoptysis among patients attending our tertiary care centre over a period of one year.

Among 60 patients studied we found that male significantly outnumbered female with male:female ratio of almost 2:1. Abal et al[5] and Fidan et al[6] also found that hemoptysis is 4.2 times more common in males than in females. The mean age of the participants was $43.93 \pm 13.20$, most of them falling into the age group of 40-59 years. All participants included in the study had only streaky or mild hemoptysis. The mean BMI was 20.16 \pm 3.26 . We observed that sputum production was the predominant associated symptom (70\%) followed by dyspnea (56.7\%), fever (33.3\%) and loss of weight(26.7\%).

In this study significant number of patients (43.3\%) were active or ex- smokers. Past history of tuberculosis was present in $43.3 \%$ of the participants. This finding is in agreement with study done by Deepal Talwar et al[7] where $54.6 \%$ of the patients with hemoptysis had previous history of being treated for TB. Thiruman et al[8] found that $90 \%$ of their study participants were active or ex smokers. Abalet al[5] also found hemoptysis in $40.4 \%$ smokers. Hence we think that previous history of tuberculosis and smoking are most important risk factors for hemoptysis.

In our research we used FOB for all patients to confirm the clinical and CXR diagnosis. On gross examination active bleeding could be localized in almost half the number of patients. Our findings are similar to other studies done in India recently. Arun BJ et al[9] conducted a study evaluating patients with hemoptysis with normal CXR and they could identify the site of bleeding in $38.89 \%$ of the cases, similarly Naveed nazir shah et al[10]- could localize bleeding site in $18.1 \%$ patients with normal CXR, $59.6 \%$ of the patients with abnormal CXR. We want to emphasize here that, localizing the bleeding site is of prime importance in these patients. FOB can be used as therapeutic tool as well, by instillation of anti bleeding agents such as cold saline, tranaxemic acid, epinephrine to control the bleeding in the same setting. Advanced modalities like electrocautery, argon plasma coagulation, laser photocoagulation etc can be used if available based on our expertise. Other gross FOB findings appreciated in our study were congestion (hyperemia), ectasia of bronchial lumen (bronchiectasis), endobronchial growth and erosion/ulcer.

Etiological pattern for hemoptysis vary in each geographical area studied. Pulmonary TB, bronchiectasis and malignancy are the most common causes of hemoptysis. The etiological order may considerably vary depending upon various factors like socio demographics and clinical profile of study 
population like age, comorbid conditions and smoking habits. It also depends on the diagnostic modalities used and time of research being conducted.

We found that bronchiectasis $(26.7 \%)$ was the most common cause followed by active pulmonary TB (20\%). Third common etiology was malignancy (16.7\%). In our study we diagnosed bronchiectasis in16 patients. Diagnosis of bronchiectasis was made based on clinical presentation, past history of pulmonary TB (present in 11 out of 16 patients), auscultatory findings supported by FOB findings of endobronchial ectasia. This result is similar to that of other studies from developing countries including India which showed an incidence of $25-35 \%$ bronchiectasis among hemoptysis patients[11,12,13]. Studies done outside india also showed a similar trend in the of bronchiectasis ranging from $20-30 \%$ in hemoptysis patients[14,15,16,17]. Active TB as a cause of hemoptysis has seen a decreasing trend in last few decades due to strengthening of diagnosis and treatment strategies under RNTCP in India[18]. We found that $20 \%$ of the patients had active pulmonary TB whereas other studies done in South India by joseph et al[12] and subodh et al[13] showed a still lower incidence of $4.68 \%$ and $8.18 \%$ respectively. Malignancy was diagnosed in $16.7 \%$ of the participants and this finding is in line with other studies done by Kapartanos et al[19] (17\%) and Ebru unsal et al[16] (18.9\%). However FOB findings were normal in 2 patients with obvious mass lesion on CXR in whom we strongly suspected malignancy. Other notable causes for hemoptysis observed in our research were bacterial pneumonia (10\%) and fungal infection (3.33\%). Among 26 patients with past history of pulmonary TB we diagnosed bronchiectasis in 11 , recurrent $\mathrm{TB}$ in 5, fungal infection in 2 patients.

The second objective of our research was to evaluate the effectiveness of FOB in establishing diagnosis in patients presenting with hemoptysis. FOB is a relatively safe and minimally invasive procedure. It precisely helps in achieving diagnosis by direct visualization of the bronchial tree and also provide microbiological and histopathological diagnosis through sampling methods like BAL and EBB. We found that FOB could establish diagnosis in two thirds of the patients with a diagnostic yield of $76.6 \%$. Uniqueness of our study is that CT thorax was not performed in our patients prior to $\mathrm{FOB}$ as $\mathrm{CT}$ will cost more to the patient than FOB at our centre. Hence we think that FOB is a very useful tool in diagnosing hemoptysis when CT thorax is not feasible in resource poor setting. This is supported by other study done in India. Navved et al[10] conclude that the diagnostic yield of FOB was $75.4 \%$ in patients with hemoptysis and localizing abnormality on CXR and $54.5 \%$ with a normal or nonlocalizing CXR.

Another aspect we want to highlight through our study is that all patients had only streaky or mild hemoptysis who were subsequently diagnosed with life threatening conditions like tuberculosis, malignancy etc. This implies that any amount of hemoptysis should be considered seriously and it does not exactly represent the severity of underlying disease. This conclusion was supported by carton et al[1] which showed that amount of hemoptysis is of less clinical significance.

\section{Strengths and limitations of the study}

Many studies done in India have evaluated the role of FOB in patients with hemoptysis as an additional investigation with CT thorax to establish the diagnosis, but none or very few studies have evaluated the etiology using FOB alone without CT thorax being performed. We believe that, this is one strength of our study. We also studied the clinical profile of these patients which help us to identify the risk factors for hemoptysis.

We used FOB for confirmation of our clinical and CXR diagnosis. Diagnosis could not be attained in $23.3 \%$ of the cases. These patients will require CT thorax for further evaluation. But we believe hemoptysis in these patients largely represent post - TB sequelae (excluding bronchiectasis). Although bronchiectasis is predominantly a high resolution CT (HRCT) diagnosis, we relied upon clinical history (like copius sputum production and past history of pulmonary TB), auscultatory findings and CXR for the diagnosis supported by FOB findings. Good bronchoscopy skills and observation to pick up ectasia of bronchial lumen on FOB in these patients is crucial. FOB couldnot pick up intrabronchial mass in two of our patients with obvious mass lesion on CXR in whom malignancy was suspected. FOB could miss peripheral lesions and the severity or extent of the disease is best assessed by CT thorax. This is a single centre study with limited sample size, the observation cannot be generalized.

Table 1: Patients clinical profile $(n=60)$

\begin{tabular}{|l|l|}
\hline \multicolumn{1}{|c|}{ Characterstics } & Mean \pm SD / number of patients (\%) \\
\hline Age in yrs, mean \pm SD & $43.93 \pm 13.20$ \\
\hline Sex, $\mathrm{n}(\%)$ & Males $-38(63.3 \%)$ \\
& Females $-22(36.7 \%)$ \\
\hline BMI, mean \pm SD & $20.16 \pm 3.26$ \\
\hline Associated Symptoms, $\mathrm{n}(\%)$ & $\begin{array}{l}\text { Sputum production }-42(70 \%) \\
\text { Dyspnea }-34(56.7 \%)\end{array}$ \\
\hline
\end{tabular}




\begin{tabular}{|l|l|}
\hline & $\begin{array}{l}\text { Fever }-20(33.3 \%) \\
\text { Loss of weight }-16(26.7 \%)\end{array}$ \\
\hline Duration of hemoptysis, $\mathrm{n}(\%)$ & $\begin{array}{l}\text { Less than 7days }-28(46.7 \%) \\
\text { more than 7 days }-28(46.7 \%) \\
\text { single episode }-04(6.7 \%)\end{array}$ \\
\hline Nature of hemoptysis, $\mathrm{n}(\%)$ & $\begin{array}{l}\text { Streaky }-18(30 \%) \\
\text { Mild }(<100 \mathrm{ml} / \mathrm{day})-42(70 \%)\end{array}$ \\
\hline $\mathrm{Co}-$ morbidities, $\mathrm{n}(\%)$ & Diabetes mellitus $-04(6.7 \%)$ \\
& Systemic hypertension $-10(16.7 \%)$ \\
& Old PTB - 26(43.3\%) \\
\hline Personal history, $\mathrm{n}(\%)$ & Tobacco smoking $-26(43.3 \%)$ \\
& Tobacco chewing $-08(13.3 \%)$ \\
& Alcohol consumption $-20(33.3 \%)$ \\
\hline
\end{tabular}

BMI - Body mass index, PTB - Pulmonary Tuberculosis, \% - percentage, SD-standard deviation, n-number of patients

Table 2: Chest $x$-ray findings

$\%$ - percentage, n-number of patients

\begin{tabular}{|l|c|}
\hline \multicolumn{1}{|c|}{ Chest $\mathbf{x}-\mathbf{r a y}$ findings } & Number of patients $(\mathbf{\%})$ \\
\hline Normal, $\mathrm{n}(\%)$ & $12(20 \%)$ \\
\hline Hilar adenopathy, $\mathrm{n}(\%)$ & $4(6.7 \%)$ \\
\hline Collapse, $\mathrm{n}(\%)$ & $2(3.3 \%)$ \\
\hline Fibrocavity, $\mathrm{n}(\%)$ & $12(20 \%)$ \\
\hline Mass Lesion, $\mathrm{n}(\%)$ & $08(13.3 \%)$ \\
\hline Ectasia, $\mathrm{n}(\%)$ & $16(26.7 \%)$ \\
\hline $\begin{array}{l}\text { Pneumonia/Unresolved } \\
\text { pneumonia, } \mathrm{n}(\%)\end{array}$ & $06(10 \%)$ \\
\hline
\end{tabular}

Table 3: Gross bronchoscopic findings

\begin{tabular}{|l|c|}
\hline \multicolumn{1}{|c|}{ FOB findings } & Number of patients (\%) \\
\hline Normal, $\mathrm{n}(\%)$ & $06(10 \%)$ \\
\hline Ectasia, $\mathrm{n}(\%)$ & $16(26.6 \%)$ \\
\hline Erosion/ulcer, $\mathrm{n}(\%)$ & $04(6.7 \%)$ \\
\hline Endobronchial growth, $\mathrm{n}(\%)$ & $08(13.3 \%)$ \\
\hline Congestion/Hyperaemia, $\mathrm{n}(\%)$ & $44(73.3 \%)$ \\
\hline Bleeding, $\mathrm{n}(\%)$ & $30(50 \%)$ \\
\hline
\end{tabular}

FOB - Fiber optic bronchoscopy, \% - percentage, n-number of patients

Table 4: BAL and EBB analysis

\begin{tabular}{|l|l|}
\hline \multicolumn{1}{|c|}{ BAL / EBB analysis } & \multicolumn{1}{c|}{ Results - Number of patients (\%) } \\
\hline BAL ZN staining/ CB-NAAT, $\mathrm{n}(\%)$ & TB detected - 12(20\%) \\
\hline BAL bacterial Culture, $\mathrm{n}(\%)$ & $\begin{array}{l}\text { Pseudomonas aeroginosa }-02(3.33 \%) \\
\text { Pneumococci }-04(6.665)\end{array}$ \\
\hline BAL Fungal elements, $\mathrm{n}(\%)$ & Positive $-02(13.3)$ \\
\hline EBB of growth, $\mathrm{n}(\%)$ & $\begin{array}{l}\text { Squamous cell carcinoma - 06(10\%) } \\
\text { Adenocarcinoma - } 04(6.7 \%)\end{array}$ \\
\hline
\end{tabular}

EBB- Endobronchialbiopsy, BAL - Bronchoalveolarlavage, ZN - ZiehlNeelson, AFB- Acid fast bacilli, CB-NAAT - Catridge based nucleic acid amplification test, \% - percentage, n-number of patients

Table 5: Final Diagnosis

\begin{tabular}{|c|c|c|}
\hline & Diagnosis & Number of patients (\%) \\
\hline & Bronchiectasis, $\mathrm{n}(\%)$ & $16(26.7 \%)$ \\
\hline & Active Pulmonary tuberculosis, n (\%) & $12(20 \%)$ \\
\hline & Bacterial pneumonia, $\mathrm{n}(\%)$ & $06(10 \%)$ \\
\hline & Fungal infection, n (\%) & $02(3.33 \%)$ \\
\hline & Squamous cell carcinoma, n (\%) & $06(10 \%)$ \\
\hline & Adenocarcinoma, n $(\%)$ & $04(6.7 \%)$ \\
\hline & Active pathology not detected, n (\%) & $14(23.3 \%)$ \\
\hline
\end{tabular}

$\%$ - percentage, n-number of patients 


\section{Conclusion}

Bronchiectasis was the most common cause of hemopytsis followed by pulmonary tuberculosis. FOB resulted in diagnosis of three-fourth of the patients analysed in our study with a positive yield of $76.6 \%$. FOB is a useful diagnostic tool in determining the etiology of hemoptysis prior to CT thorax.

\section{References}

1. Carlton R, Anne ST. The Clinical significance of hemoptysis. N Eng J Med 1952;247:790-3.

2. Khedral I, Braun SR. Haemopytsis. In: Braun SR (Ed) concise textbook of pulmonary medicine.1st Edn, New York : Elsevier Since Publishing Co;1989;1:603-8.

3. American Thoracic Society. The management of haemoptysis. Am Rev Respir Dis 1966;93:471-4.

4. Weinberger SE, Braunwald E. Cough and hemoptysis. In: Braunwald E, Fauci AS, Kasper DL, Hauser SL, Longo DL. Harrison's principle of internal medicine. $15^{\text {th }} \mathrm{edn}$, McGraw Hill:2001.p.203-7

5. Abal AT, Nair PC and Cherian J. Haemoptysis: Aetiology, evaluation and outcome a prospective study in a third world country. Respir Med 2001;95:548-52.

6. Fidan A, Ozdogan S, Oruc O, et al. Hemoptysis: A retrospective analysis 108 cases. Respir Med 2002;96:677-80.

7. Deepak Talwar, JiteshChudiwal, RC Jain, Sandeepan Kumar. Haemoptysis: causes, interventions and outcomes-Indian single centre experience. Eur Respirat Soc 2012;40:3491.

8. M Thirumaran, R Sundar, IM Sutcliffe, DC Currie. Is investigation of patients with haemoptysis and normal chest radiogragh justified? Thorax 2009;64:854-6.

9. Arun B. J, Mohan J, Vijay Bhaskar, Niranjan Nagaraj. Role of fiberoptic bronchoscopy in cases of hemoptysis with normal chest radiograph. Int J Adv Med 2016;3(3):691-3.

10. Naveed Nazir Shah, Manzoor Ahmad Wani, Syed Quibtiya Khursheed, Rakesh Bargava, Zuber Ahmad, Khurshid Ahmad Dar et al. Role of fiberoptic bronchoscopy in haemoptysis: an analysis of 157 patients. Int J Res Med Sci 2015;3(9):2386-93.
11. Rao PU. Hemoptysis as a symptom in a chest clinic. Indian J Chest Dis 1960;2:219.

12. Joseph T, Nair S, James PT (2017) Clinical-Radiological, Pathological Profile and Treatment Outcome of Patients with Haemoptysis. J Pulm Respir Med 2017;7:437.

13. Subodh K. Nawal, Mamta R. Heda. Hemoptysis: A Prospective Analysis of 110 Cases. Asian J Biomed Pharmaceut Sci 2013;3(21):1-3.

14. Hirshberg B, Biran I, Glazer M, Mordechai R. Haemoptysis: aetiology, evaluation and outcome in a tertiary referal Hospital. Chest 1997; 112: 440-4.

15. McGuinness G, Beacher JR, Harkin TJ, et al. Haemoptysis: prospective high resolution CT/bronchoscopic correlation. Chest 1994;105:1155-62.

16. Ebru Unsal, Deniz Koksal, Filiz Cimen, Taci Hoca, Tugrul Sipit. Analysis of patients of hemoptysis in a reference hospital for chest disease. Tuberkuloz Ve Toraks Dergisi 2006;54(1):34-42.

17. Jin-Fu Xu, Xiao-Bing J, Hu-Ping, Hai-Wen Lu. Bronchiectasis single centre study. ERS J 2013;42:7.

18. Chauhan LS, Tonsing J. Revised national TB control programme in India. Tuberculosis (Edinb) 2005;85(56):271-6.

19. A Kapartanos, E Argyropoulou, F Sampsonas, A Zania, M Tsiamita, K Spiropaulos. Indications, results and complications of flexible fiberopticbronchoscopy: a 5 year experience in a referral population in Greece. Eur Rev Med Pharmacol Sci 2008;12:355-63.

How to cite this article: Bondade K, Ajit E, Kulkarni H. K, Dacosta A. L, Pinto M. Clinico-etiological profile of patients presenting with hemoptysis diagnosed by fiber optic bronchoscopy - A study done in tertiary care centre of central Karnataka. Indian J Immunol Respir Med. 2018;3(4):188-192. 